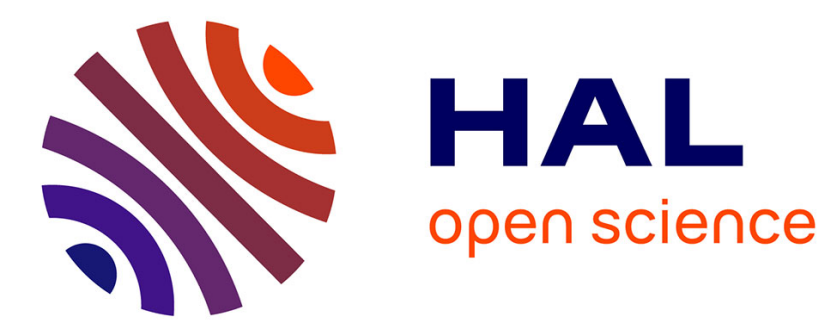

\title{
Un milliaire de Philippe l'Arabe à la frontière des Rutènes et des Gabales
}

Michel Labrousse

\section{To cite this version:}

Michel Labrousse. Un milliaire de Philippe l'Arabe à la frontière des Rutènes et des Gabales. Gallia - Fouilles et monuments archéologiques en France métropolitaine, 1980, 38 (2), pp.247-251. 10.3406/galia.1980.1800 . hal-01936222

\section{HAL Id: hal-01936222 \\ https://hal.science/hal-01936222}

Submitted on 27 Feb 2020

HAL is a multi-disciplinary open access archive for the deposit and dissemination of scientific research documents, whether they are published or not. The documents may come from teaching and research institutions in France or abroad, or from public or private research centers.
L'archive ouverte pluridisciplinaire HAL, est destinée au dépôt et à la diffusion de documents scientifiques de niveau recherche, publiés ou non, émanant des établissements d'enseignement et de recherche français ou étrangers, des laboratoires publics ou privés.

\section{(ㅇ)(1) $\$$}

Distributed under a Creative Commons Attribution - NonCommercial - NoDerivatives| 4.0 


\title{
UN MILLIAIRE DE PHILIPPE. L'ARABE A LA FRONTIÈRE DES RUTÈNES ET DES GABALES
}

\author{
par Michel LABROUSSE
}

Depuis 1977, M. P.-M. Blanquet, animateur du Centre d'études et de recherches du milieu rural de Campagnac, poursuit, sur les pentes ouest de la butte du château de Sévérac, des prospections $^{1}$ qui attestent une occupation du site depuis les débuts de l'Age du Fer jusqu'au Moyen Age et au-delà2. Dans les premiers jours d'août 1979, il prévenait M. Alain Vernhet, directeur des fouilles de La Graufesenque, que venait d'être signalé près du Villaret, sur la limite est de la commune de Sévérac, un milliaire romain avec inscription latine. Dès le 15 août, nous nous sommes rendus sur place, en compagnie de M. Blanquet, de toute l'équipe des fouilleurs de La Graufesenque et des membres de l'Association pour la sauvegarde du patrimoine caussenard que préside à Sévérac le général Roubertier. Sous une pluie battante, le milliaire, taillé dans un calcaire

1 En publiant ces quelques pages, je dois exprimer ma gratitude à tous ceux qui ont contribué à la découverte et à l'étude première du milliaire du Villaret, à M. P.-M. Blanquet, à M. A. Vernhet et à tous les fouilleurs de La Graufesenque, au général Roubertier et aux membres de l'Association pour la sauvegarde du patrimoine caussenard, à qui nous devons la découverte toute récente, près de Villeplaine, commune de Sévérac, d'un tumulus à inhumation des débuts de l'Age du Fer et, dans la même commune, les premières fouilles des établissements gallo-romains d'Altès.

2 Sur ces premières découvertes, cf. Gallia, 36, 1978, p. 396. local, a été retrouvé, à $500 \mathrm{~m}$ au nord-est du Villaret, renversé sur un chicot calcaire qui domine une luzerne appartenant à M. Gaston André, de Mostuéjouls ${ }^{3}$.

D'un diamètre de $0,30 \mathrm{~m}$, sa partie supérieure, conservée sur une hauteur de $0,35 \mathrm{~m}$, gardait trois lignes encore lisibles, profondément gravées en lettres de $0,06 \mathrm{~m}$ et de $0,05 \mathrm{~m}$ (fig. 1).

$$
\begin{aligned}
& \text { IMP.GAES } \cdot M \cdot I V L \\
& \text { PHILIPPO } \cdot \text { ET } \cdot M \cdot I V L I O \\
& \text { P.... NOBILISSI } . \\
& \ldots \ldots \ldots \ldots \ldots \ldots \ldots
\end{aligned}
$$

La cassure de la partie inférieure paraissait de fraîche date et, de fait, M. Georges-André Colas, découvrait, à quelques mètres de distance, le fragment initial de la troisième ligne portant la lettre P..... de P[HILIPPO]. De nouvelles recherches permettraient peutêtre de rassembler d'autres fragments du milliaire.

3 La découverte se situe au lieu-dit Larinié, dans la parcelle 6 de la section YB du cadastre rénové de Sévérac. Coordonnées Lambert (zone III) sur la carte au 1/25 000, feuille de Sévérac-le-Château, n ns 7-8 : 664.925-225.950; altitude : $810 \mathrm{~m}$. Le lieu est pratiquement à cheval sur la faille qui sépare les calcaires sinémuriens du lias $\left(1^{2}\right)$ de la dépression de Sévérac et les calcaires jurassiques séquaniens-rauraciens ( $\left.\mathrm{J}^{4-3} \mathrm{~b}\right)$ des Causses de Sévérac et de Sauveterre. 

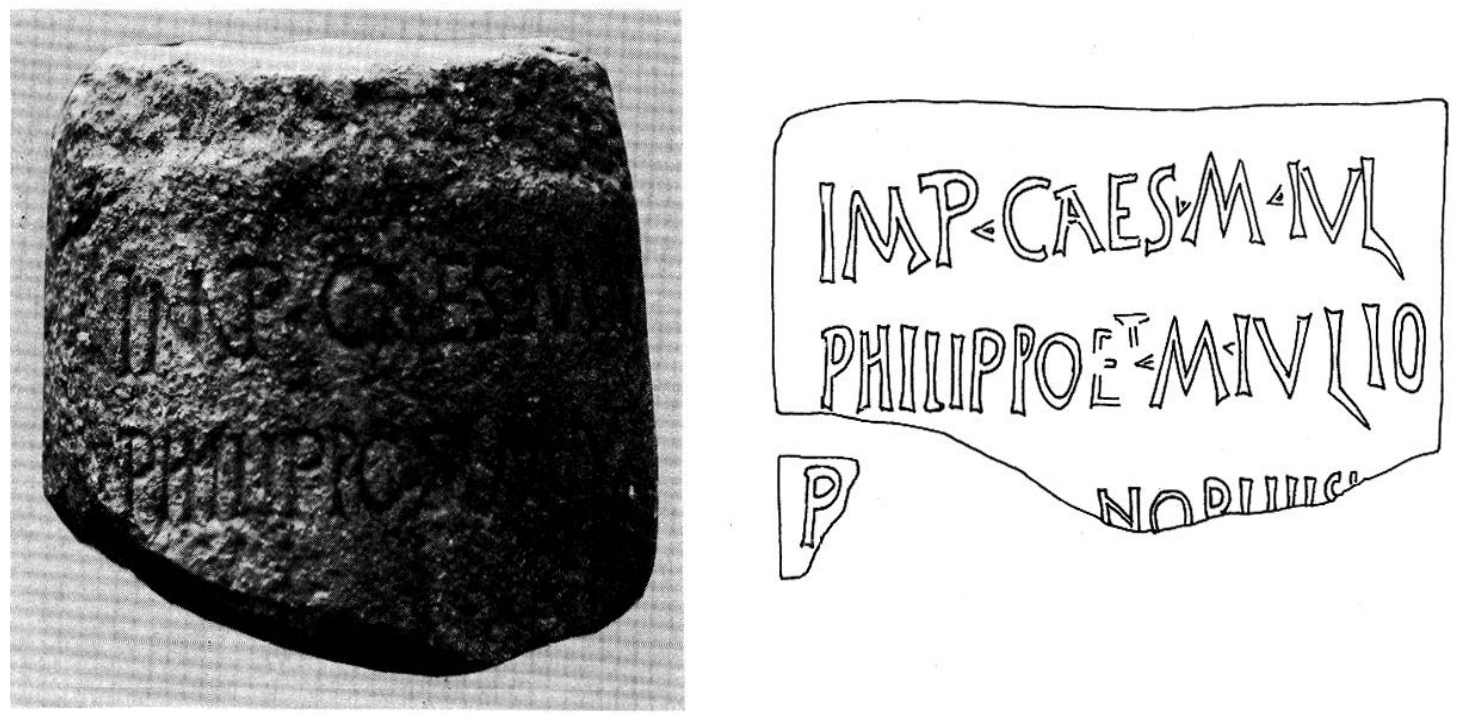

1 Le milliaire du Villarel. Calcaire (haut. : $0,35 \mathrm{~m}$ ).

Sa lecture est, de toute façon, certaine :

Imp(eralori) Caes(ari) M(arco) Iul(io) I Philippo et $M($ arco) Iulio / P[hilippo], nobilis $[$ simo / Caesari $]$......

"A l'empereur César M. Iulius Philippus et à M. Iulius Philippus (son fils), très noble César...... ".

Il s'agit d'un milliaire de l'empereur Philippe dit l'Arabe ${ }^{4}$, dont le court règne s'inscrit entre 244 et 249.

Huit milliaires de Philippe étaient déjà connus en Gaule 5 . Quatre viennent de provinces très éloignées du Rouergue : extrême

4 Il doit ce nom, sans caractère officiel, au fait que sa famille, de condition équestre comme lui-même, était originaire de la Trachonitide dans la province d'Arabie, où il fonda la ville de Philippopolis (auj. Shahba, au nord du Djebel Druse, en Syrie).

5 Liste dans Jullian, Histoire de la Gaule, IV, p. 556, n. 3, à compléter par le milliaire de Champlieu (Wuilleumier, Inscriptions latines des Trois Gaules, XVII' suppl. à Gallia, 1963 (abr. I.L.T.(i.), no 484). - En dehors des milliaires, deux dédicaces en l'honneur de Philippe ou des membres de sa famille ont été gravées à Vence et à Béziers (C.I.L., XII, 10 et 4227) et deux tauroboles ont été célébrés pour le salut de l'empereur el des siens à Die et à Nîmes (C.I.L., XII, $1567 ;$ A.E., $1910,217=$ A.E., 1924, $26=$ EsPर́RANDIEU, Inscr. Latines de la Gaule Narbonnaise, $\mathrm{n}^{\circ} 518$ ). nord de la Narbonnaise, Germanie Supérieure, Gaule Belgique.

Aux confins de la Narbonnaise et de la Germanie, un milliaire a été retrouvé sur la route qui, de Nyon à Genève, longeait la rive nord du Léman ${ }^{6}$. Deux autres jalonnaient, en Germanie Supérieure, la grande voie militaire qui unissait Mayence à la Rétie par la rive droite du Rhin et la vallée du Neckar?. En Gaule Belgique, à Champlieu, au s. de la forêt de Compiègne, un milliaire de Philippe, associé à des milliaires de Gordien III, de Trébonien Galle et de Dioclétien, devait marquer, vingt lieues gauloises à l'ouest de Soissons, la frontière des Suessions et des Silvanectes ${ }^{8}$.

Dans la Grande Aquitaine augustéenne dont dépendait le Rouergue, un milliaire de Philippe a été signalé à l'extrême sud, à Labroquère (Haute-Garonne), à 1,5 km de Saint-Bertrand de-Comminges, chef-lieu des Convènes. Il appartenait au tronçon commun des voies romaines qui allaient au nord vers Agen et au

6 C.I.L., XII, 5531.

7 C.I.I., XIII, 9100 et 9108 : milliaires de Ladenburg et d'Heidelberg.

8 Wullteumier, I.L.T. (i., $\mathrm{n}^{\circ} 484$; cf. F. AirberTINI, Milliaires de la route romaine de Senlis à Soissons, dans Comptes rendus de l'Académie des Inscriptions et Belles Lettres, 1919, p. 46-53 ; carte dans Albert GreNiER, Manuel..., II, 1, p. 179, fig. 56. 
nord-est vers Toulouse ${ }^{9}$. Trois autres ont été retrouvés, beaucoup plus près du Rouergue, sur les grandes routes qui, partant de Lyon, traversaient le Massif Central : un à Vichy, sur un itinéraire Lyon-Clermont ${ }^{10}$ et surtout deux dans le Velay, à l'ouest de Saint-Paulien, capitale des Vélaves, sur la transversale de Lyon à Bordeaux par le sud du Massif Central, Javols, Rodez et Cahors ${ }^{11}$.

Comparé à ces milliaires, celui du Villaret offre une titulature simplifiée de Philippe : il supprime le prédicat $D$ (ominus) $N$ (osler) commun à ceux du Velay et de Vichy, ainsi que les titres officiels de $P$ (ius) F(elix) Aug(ustus), pourtant partout présents sauf à Vichy et à Champlieu. En outre, selon une règle générale, qui ne souffre d'exception qu'à Labroquère, il ne parait pas associer au nom de l'empereur et de son fils celui de l'impératrice Marcia Otacilia Severa.

Pour le Rouergue, il vient s'ajouter à la liste très courte des quatre milliaires recensés en 1948 par Alexandre Albenque, liste qui ne s'était pas enrichie depuis ${ }^{12}$ : le milliaire de Trébonien Galle et de Volusien (251-253) retiré vers 1880 des ruines de l'église Saint-Vincent de Rodez, qui, selon l'hypothèse d'Albenque, aurait appartenu à la grande transversale de Lyon à Bordeaux, jalonnée en pays vellave par les milliaires de Philippe $^{13}$; - la borne des Enfruts, commune de Saint-Chély-d'Aubrac,

9 J. SACaze, Inscr. antiques des Pyrénées, Toulouse, 1892 , p. $215-217, \mathrm{n}^{\circ} 147=$ C.I.L., XIII, 8889 ; cf. R. Lizop, Les Convenae et les Consoranni, Paris et Toulouse, 1931, p. 114, n. 1 ; Michel Labrousse, dans l'Histoire de la Gascogne, publiée sous la direction de Maurice Bordes, Roanne, 1977, p. 41.

10 C.I.L., XIII, 8905 ; la dislance porlée sur la borne est de 21 lieues gauloises comptées à partir de Clermont, chef-lieu de la cilé des Arvernes.

11 C.I.L., XIII, 8873 et 8878 ; sur les bornes, retrouvées hors de place à Solilhac et à Sanssac, les distances portées sont de 3 et de 6 milles complés à partir de Saint-Paulien (Ruessio).

12 Sur les milliaires du Rouergue, en dernier lieu, Michel Labrousse dans Hisloire du Rouergue, publiéc sous la direction d'Henri Enjalbert, Toulouse, 1979, p. $46-47$.

13 C.I.L., XIII, 8885 ; cf. A. A I.BENQUE, Inventaire de l'archéologie gallo-romaine du département de l'Aveyron, Rodez, 1947, p. 117 (no 310) et 196, Les Rulènes, Roder, 1948, p. 289-291. sur la route de Rodez à Javols, autre section de la même transversale ${ }^{14}$; - le milliaire de Foncavette, au nom de Constance Chlore, découvert en 1920 près de Vailhourles, sur un raccourci entre Carantomagus (Cranton) et la station de Cosa, près d'Albias, en Bas-Quercy ${ }^{15}$; - la borne, aujourd'hui disparue, du pont de la Monnaie, près d'Anglars, sur la route de Rodez à Limoges et à Périgueux qui allait passer le Lot à gué sous Capdenac ${ }^{16}$.

Cinquième de la série, le milliaire du Villaret a son originalité. A $6 \mathrm{~km}$ à l'est de Sévérac, il se dressait hors de toutes les routes principales portées sur la Table de Peulinger et de toutes les routes secondaires reconnues ou soupçonnées par l'archéologie locale ${ }^{17}$. Au pied des escarpements du Causse de Sauveterre, le Villaret et ses environs font un peu effet de cul-de-sac où nul, pas même Albenque, pourtant originaire du pays, ne pouvait deviner une voie antique. Le milliaire vient affirmer son existence. Relief et topographie semblent exclure pour elle une orientation d'est en ouest. Plus plausible est une direction générale nordsud, proche de l'axe que définissent aujourd'hui, du Lot et de la R.N. 9 au Massegros dans la Lozère, le C.D. 67, puis, du Massegros à Boyne sur le Tarn, le G.D. 32 dans la Lozère et son prolongement, le C.D. 9 dans l'Aveyron (fig. 2).

Un itinéraire comparable ou plutôt des itinéraires comparables ont existé dès l'Antiquité. Dressant en 1934, sur les données de son collègue le géographe Baulig, une carte des drailles et des chemins de troupeaux aux confins du Gévaudan et du Rouergue, Albert Grenier a marqué un chemin continu qui partait de Banassac sur le $\operatorname{Lot}^{18}$, allait droit au sud par

14 G.I.L., XIII, 8884 ; cf. A. Alßenque, Inventaire..., p. 134 (no 376 ) et 196, Les Rutènes, p. 143, 144 et 293 .

15 A. Albenque, Invenlaire..., p. 154 (no 488) el 198, 199, Les Rutènes, p. 151, 152.(fig. 11) et 291-293.

16 A. Albenque, Inventaire..., p. 47 (no 7) et 198, Les Rutènes, p. 150-151.

17 Sur ces routes, cf. A. A nzenQue, Inventaire..., p. 195-201; voir aussi, Michel lankousse, op. cil., p. 46-47, ct carte, p. 56, fig. 4 .

18 Albert Grenier, Manuel..., II, 1, p. 163, fig. 52 ; pour le détail, se reporter à la Carte de France au $1 / 50000$, feuille de Sévérac-le-Château. 
Saint-Saturnin ${ }^{19}$, un Villaret différent du nôtre, le $\mathrm{Cros}^{20}$, le Duc ${ }^{21}$, le Bétonès ${ }^{22}$ et allait rejoindre à la Bastide, à $4 \mathrm{~km}$ au nord du Massegros, l'actuel C.D. 32.

La position du milliaire du Villaret de Sévérac suggérerait un tracé plus proche, vers l'ouest, de la limite du Rouergue. Se confondaitil, plus ou moins, avec celui de l'actuel C.D. 67 par la Tieule et le Recoux ou se rattachait-il au précédent par une bretelle allant du Duc au Recoux? L'hésitation est permise.

Le milliaire a été retrouvé à $1 \mathrm{~km}$ au sud du Recoux. La fraîcheur de son inscription indique qu'il n'est pas longtemps resté exposé aux intempéries, ni dans l'Antiquité, ni de nos jours. Il a dû être enfoui dès 249 , lors de la disparition de Philippe l'Arabe ${ }^{23}$, et il a certainement été brisé et jeté en contrebas du chemin antique, il y a peu de mois, par les engins mécaniques travaillant au remembrement et à l'élargissement du chemin.

Celui-ci s'élève immédiatement vers le sud, le long de l'escarpement du Causse de Sauveterre, en direction de la cote 804 , de la bergerie située à $0,5 \mathrm{~km}$ à l'est de Corbières (cote 906), recoupant, à $1,5 \mathrm{~km}$ à l'ouest du Massegros, la R.N. 59521. A ses débuts tout au moins, il n'a qu'une largeur de 3 à $4 \mathrm{~m}$, convenable pour des files de chevaux ou de mulets, malaisée pour des attelages. La tradition locale en fait un chemin saunier, par où le sel de la Méditerranée aurait été porté en ces confins lointains du Rouergue et du Gévaudan ${ }^{25}$. Son

19 Commune du canton de La Canourgue (Lozère).

20 Hameaux de la commune de Saint-Saturnin, situés respectivement à $0,6 \mathrm{~km}$ au sud-sud-ouest et à $\mathrm{l}, 6 \mathrm{~km}$ au sud du village. Ce Villaret est à distinguer de celui de la commune de Sévérac d'où provient le milliaire de Philippe.

21 Hameau de la commune de la Tieule, canton de La Canourgue (Lozère), à $2,2 \mathrm{~km}$ à l'esl du village.

22 Hameau de la commune du Recoux, canton du Massegros (Lozère), à $3,2 \mathrm{~km}$ au nord-nord-est du village.

23 Philippe disparut, fin septembre 249, lors de sa défaite, près de Vérone, par les armécs de Dèce.

24 Voir la Carte de France au 1/50000, feuille de Sévérac-le-Château.

25 Sur les chemins sauniers el les voies romaines, cf. Albert Grenier, Manuel..., II, 1, p. 164-169.

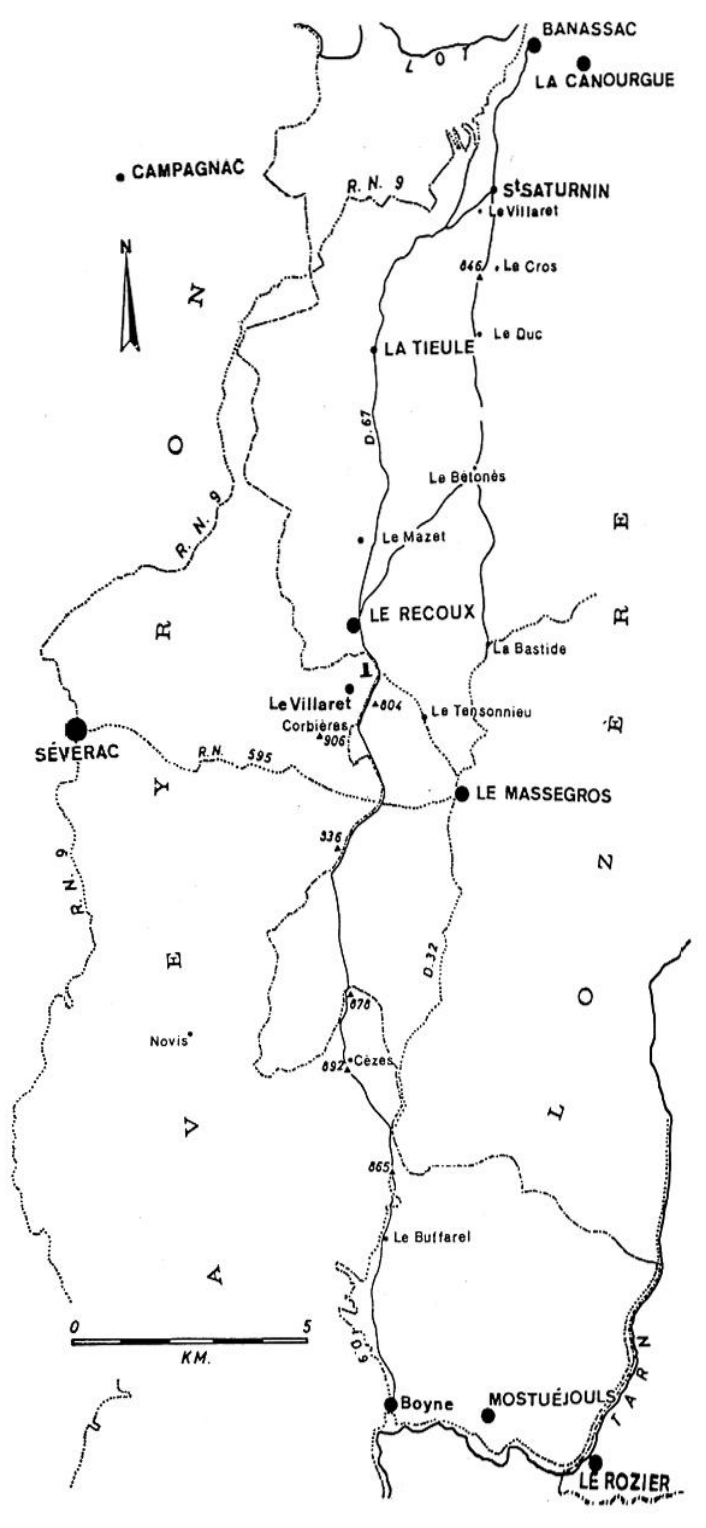

2 Itinéraires anciens entre Lot et Tarn.

origine antique semble confirmée par le fait qu'il sert de limite aux départements de l'Aveyron et de la Lozère, frontière séculaire des Rutènes et des Gabales. Une suite de chemins de terre et de pistes ${ }^{26}$ parait marquer sa continuation vers le sud par les cotes 936 ,

26 Voir, pour le délail, la Carte de France au $1 / 50000$, feuilles de Sévérac-le-Châleau et de SaintBeauzély. 
878, 892, Cézes et le Buffarel ${ }^{27}$ avant une descente à Boyne dans la vallée du Tarn ${ }^{28}$.

Dans les parties hautes, relativement peu accidentées du Causse de Sauveterre, pareil itinéraire a dû offrir, au cours des âges, des variantes nombreuses et changeantes. Sa direction générale ne fut pourtant jamais oubliée et, en 1754, lorsqu'il fut question d'ouvrir la route royale entre Aguessac et Banassac, l'un des tracés proposés ${ }^{29}$ passait par Boyne, le Tensonnieu et le Mazet ${ }^{30}$, ces deux derniers points se situant respectivement à $1,5 \mathrm{~km}$ au sud-est et à $2,1 \mathrm{~km}$ au nord de l'emplacement du milliaire de Philippe.

A l'ouest du Villaret, ont été reconnues de longue date les traces d'un ou de plusieurs grands domaines gallo-romains ${ }^{31}$. Ce sont probablement leurs propriétaires qui ont fait graver et dresser le milliaire. Ils tenaient

27 Cézes et le Buffarel sont compris dans les limites de la commune de Mostuéjouls (Aveyron). Près du Buffarel, en bordure précisément du C. D. 9, a été signalé un établissement gallo-romain marqué par la présence de nombreuses tuiles à rebord (A. Albengue, Inventaire..., p. 95, n²12).

28 Boyne dépend de la commune de Rivière-surTarn (Aveyron); dans l'église est conservé un cippe funéraire gallo-romain avec épitaphe, réutilisé comme bénitier (A. Albenque, Inventaire..., p. 106, n 268, Les Rutenes, p. 295-296 et pl. XI, no 3).

29 A ce tracé fut préférée la route actuelle par Sévérac qui ne fut, d'ailleurs, ouverte à la circulation qu'en 1841.

30 Gf. H. Guilhaumon, dans son édition du Journal des voyages en Haute-Guyenne de J.-F. Henry de Richeprey, Rodez, 1952, p. 78, n. 2.

31 Cf. A. Albenque, Inventaire..., p. 150, nos 462, 463, Les Rutènes, p. 247-248 et fig. 20. à affirmer leur loyalisme romain en honorant tous les empereurs, si éphémères fussent-ils ${ }^{32}$. Intentionnellement, ils l'ont placé en un point caractéristique de la voie, à la frontière même des Rutènes et des Gabales, comme le milliaire contemporain de Champlieu l'était à celle des Suessions et des Silvanectes ${ }^{33}$.

En ce milieu du III $^{\mathrm{e}}$ siècle, la voie avait sans doute beaucoup perdu de son importance économique, ce qui pourrait expliquer son effacement $^{34}$. Par contre, deux siècles plus tôt, du Tarn au Lot, elle avait assuré les relations les plus directes entre les trois grands centres rutènes et gabales producteurs de céramiques sigillées, La Graufesenque, le Rozier et Banassac. Tout autant que l'échange des marchandises, elle avait aidé celui des techniques, des modèles et des thèmes décoratifs. Le milliaire du Villaret apparait ainsi comme le témoin attardé d'une route, jusqu'ici inconnue, mais qui, auparavant, avait contribué plus qu'une autre à une certaine unité de l'industrie céramique du Sud de la Gaule.

Michel Labrousse.

32 L'hypothèse d'une réparation ou d'une reconstruction de la voie n'est pas à exclure, mais elle ne joua sans doute qu'accessoirement par rapport à l'intention politique. Le milliaire est, en fait, comme bien d'autres, une dédicace à l'empereur.

33 Voir ci-dessus, p. 248 et n. 8.

34 Les monnaies les plus récentes trouvées à La Graufesenque, dans le chantier du champ Cluzel, sont de Gordien III (238-244); à cette date, la production de la céramique sigillée avait cessé (cf. Michel Labrousse, Gallia, 32, 1974, p. $460 ; 34,1976$, p. 465). 